head of the humerus, the elbow being at the same time drawn backwards. In this manner, with hardly any force, the head of the humerus returned to the glenoid cavity with an audible saap. 'The patient, although over seventy years of age, has had no unfavourable symptom since the accident.

\section{syltedical Sorieties.}

\section{ROYAL MEDICAL AND CHIRURGICAL SOCIETY.}

Tuesday, APRM 14TH, 1874.

Dr. C. J. B. Williams, F.R.S., President, in the Chair.

THE ETIOLO§Y OF BRIGHT'S DISEASE AND THE PREALBUMINURIC STAGE.

BY F. A. MAHOMED

RESTDENT MTDICAT OFTICTR OF THB LONDOX PEVER FOSPITAT.

(Communicated by W. H. BROADBHNT, M.D.)

Two symptoms are described which indicate a condition of high tension in the arterial system-viz.: 1. Sphygmographic tracings, which possess the following characteristics: (a) considerable pressure required to extinguish the pulse; (b) prolongation and undue sustension of the tidal wave; (c) well-marked percussion; (d) small amount of dicrotism. 2. A blue reaction obtained with the guaiacum test for blood, if delicately applied, when no albumen or blood cells can be discovered by the microscope, spectroscope, or nitricacid test. The mode of procedure, having previously tested reagents and paper employed, is as follows: (a) dip a slip of white blotting-paper in the urine to be examined; $(b)$ evaporate the urine by drying the slip over a spirit-lamp (c) add a drop or two of tincture of guaiacum, and allow time for evaporation; (d) add a drop or two of ozonic ether, and allow the paper to become thoroughly dry before the reaction is judged of.

It is then shown from cases of so-called " inflammatory Bright's disease," occurring during the convalescence from scarlatina, that the series of pathological events occur in the following order:-1. A poisoned condition of blood: by uric acid or other efiete material-as in scarlatina, measles, erysipelas, pregnancy, or a severe chill. 2. Arrest of the action of one of the excretory organs. 3. Increase of tension in the arterial system. 4. Transudation of the crystalloids of the blood through the kidney. (These conditions constitute the prealbuminuric stage, and are easily remediable.) 5. Albuminuria; followed by dropsy and the usual symptoms of Bright's disease. 6. Changes in the kidney and other excretory organs - namely, intestinal tract and skin-produced by acute, and afterwards prolonged, congestion and high arterial tension-viz., (1) exudation and plugging of tubules or follicles; (2) fatty degeneration and absorption; (3) contraction.

Puerperal eclampsia and albuminuria are accounted for by the predisposing condition of high tension in the arterial system which exists during pregnancy. Poisons-such as gout, lead, or alcohol-produce a similar but less intense condition of increased arterial tension and excretory congestion; they also produce chronic changes, though of a different character, their onset being gradual, and not sudden. The first two changes of the acute conditionnamely, exudation and plugging of tubules, followed by fatty degeneration and absorption-do not exist; they may, however, at any time be produced, and symptoms of the first stage then supervene. The name "inflammatory Bright's disease" is discussed: the changes are ascribed to "congestion under high pressure"; while a condition of inflammation is denied. The term "Bright's disease" is considered too indefinite. A suggestion is made that the terms "acute" and "chronic excretory congestion" are less erroneous than those in general use; while the amyloid and true fatty kidneys are always included in the forms of degeneration to which they each belong.

Dr. BROADBENT said he could bear witness to the fact brought forward in the paper, as he had seen the investigation. He regretted that the extent of the bluing could not be shown as well as the tracings. He had seen it daily, and it was a great proof of the accumalation of the debris in the blood. The paper was of interest physiologically and clini- cally: physiologically as showing the relation and cause of the high blood tension, which was secondary to capillary resistance and defensive; and clinically it showed the benefit from treating early threatening albuminuric complications. The prealbuminuric stage was detected, and removed either by purgation or the hot pack.

Dr. Dickinson did not agree with the statement that other organs were affected before the kidney in albuminuria from scarlet fever; he thought the kidneys deserved the priority. As to the use of purgation in cases of scarlet fever and dropsy, he had seen many cases set in in which there had been no constipation. One point was difficult to him. Mr. Mahomed would put both forms of Bright's disease, the inflammatory and fatty, and the granular, under "excretory congestion." It was hard to class two opposite diseases under the same term. He agreed that the inflammatory condition from measles and scarlet fever was preceded by blood-poisoning, but the granular kidney depended upon other causes; it may be hereditary, and appear early. He knew three members of one family who died of the granular kidney, and there was no blood-poisoning there. It was often associated with fibroid change in the arteries.

Dr. BRUNTon said the paper drew attention to the resistance to the blood in Bright's dicease, and the part it played in producing albuminuria. Albumen has been ascribed to the blood and the kidney. There is no proof that it depends upon a modification of the first, so we must look to the kidneys. Here there are two structures, the vessels and the epithelium lining the tubules. Disease of either produces albuminuria; by some it is said to be always due to the epithelium, but it is not so. So the resistance in the vessels is the cause, and change of pressure will bring on albuminuria. Increased high tension will not always do so, for there may be high tension elsewhere and low tension in the vessels of the kidney, because they contract, regulating the supply of the blood. The paper showed how to diagnose high tension before it reached a dangerous point, and so we were able to relieve it.

Dr. Sibson said the paper was valuable for the facts it contained; we wanted facts without inferences. The paper showed that in scarlatina, before albuminuria set in, there was distinct arterial tension, recognised by the escape of crystalloids into the urine, and that by purging the arterial tension was lessened; but he did not think we could say that the purging prevented albuminuria. He did not think it well to group the granular kidney with the other forms, nor did he like to alter a nomenclature for the sake of an opinion. Why should thé name of Bright's disease be given up?

Dr. MILner Fothergill said albuminuria was asisociated with high arterial tension, and alluded to Frerichs' experiments. He thought there was some alteration in the condition of the blood inducing this high tension. The arterial resistance being increased, the left ventricle was hypertrophied. He thought the high tension had something to do with the condition of kidney described.

'The President said the subject was exceedingly interesting ; in all cases of high tension before albumen was found in the urine, the crystalloid substances became apparent. He demurred to the extent of the importance given to high tension. In all cases of high tension the urine was scanty and high-coloured and altered before albumen appeared. The tense cord-like pulse and the reduplication of the first sound of the heart, in cases of albuminuria and diseases of the heart and kidney before the albumen was pronounced, had of ten led him to say it would occur. The reduplication depended upon the condition of the blood from retained excrementitious matter, which increased the arterial resistance throughout the system. After purging-salines and digitalis-the arterial resistance disappeared. Albumen often arose from congestion, but congestion must go on to altered structure before we have kidney disease. He thought albuminuria was more likely to arise from disease of the kidney than from altered tension of the blond.

Mr. Maнomed said Dr. Dickinson and Dr. Sibson considered he contonisded the large white kidney with the cirrbotic. He did not mean to do so, or to infer that the white passed into the cirrhotic. He believed the gouty contracted kidney began as such. He used the term excretory enngestion as acute -and chronic, putting the cirrhotic kidney into the chronic. The contracted kidney 
was said to be hereditary, but it could not be born so; there must be some change in the blood producing blood-poisoning; the atheroma was the result of high tension. He had used the name excretory congestion because all the excretory organs, skin, \&c., were affected. There was a distinction between albumen from venous congestion and arterial alteration; in venous albuminuria there was no guaiacum test, but in arterial albuminuria it was always present. With the assistance of Dr. Brunton, he had made the following experiment. A large dog was chloroformed and the urine tested; there was no guaiacum reaction. The aorta was then ligatured below the renal vessels; the next urine drawn gave the blue reaction, and was alkaline. In albuninuria from lead-poisoning, as the lead is removed the albumen disappears. The urine before albuminuria was always scanty, as far as he had observed.

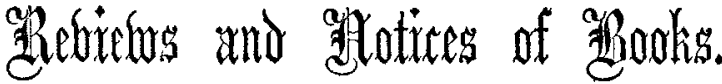

Diseases of the Urinary Organs, including Stricture of the Urethra, Affections of the Prostate, and Stone in the Bladder. By John W. S. Goulex, M.D., late Professor of Clinical Surgery and Genito-Urinary Diseases in the Medical Department of the University of the City of New York; Surgeon to Bellevue Hospital, \&c. With 103 Wood Bngravings. pp. 368 . New York: W. Wood and Co.

London : Trübner. 1873.

The author of this work occupies, we believe, a high position in New York as an authority on genito-urinary disorders, and we are glad, therefore, to have the opportunity of comparing the views he entertains with those held on this side of the Atlantic. Dr. Gouley is evidently fully aware of all that is being done in regard to his special department both in this country and on the continent, and it is interesting to find that some of the conclusions he bas arrived at are just the reverse of those which have of late been somewhat persistently urged upon the profession here. Thus Dr. Gouley emphatically lays down (p. 25) that gum. elastic catheters do not give less pain to the patient than metal instruments, and he himself always employs metal sounds for the larger sizes-i.e., above No. 7 ; and he says: "Patients, in taking note of the change of instrument, almost always speak of the slight pain they feel during the passage of the metallic, as compared with that produced by the gum instrument." Dr. Gouley also prefers the recumbent position for the patient upon whom a catheter is being passed.

Apropos of some recent teaching, we may quote the following (p. 43) :-

"The surgical treatment is demanded in all the stages of stricture, and without it internal medication is of little avail. This already well-known fact is here stated because there are practitioners who still entertain the idea that if patients suffering from obstructed micturition due to stricture be at once put to bed, and treated medicinally, or even expectantly, they will soon get well. In answer to this, it may be said that when patients make a good recovery under such treatment, they have not been suffering from stricture, but probably from temporary obstruction in the deeper part of the urethra, caused by inflammatory swelling, which will frequently subside by rest alone. Those who believe that they cure stricture in this way do not usually resort to urethral exploration, without which no diagnosis can be accepted as conclusive."

In cases of tight stricture the author prefers slender probe-pointed whalebone bougies, over which he slides his own "tunneled sound" or an elastic catheter, thus following Mr. Wakley's practice. Treatment of stricture by caustics and electrolysis are condemned, but the author has a high opinion of "divulsion," or "splitting," as practised by Mr. Holt. On this subject Dr. Gouley says (page 61) : "Extensive experience has taught the profession that nothing short of complete divulsion of the stricture will ensure success, and that, after all, it amounts to about the same thing as an internal urethrotomy; but it is a safer operation. Therefore Sir Henry Thompson's precept of stretching as much and tearing as little as possible might well be reversed; and I will venture to say, tear as much and stretch as little as possible, and the results will be all the better." Again, at page 83, the author says: "My own preference is now for divulsion, and $I$ only resort to internal urethrotomy in intractable strictures of the ante-scrotal portion of the canal."

Both internal and external urethrotomy are recommended by Dr. Gouley in suitable cases, and he has contrived a special grooved and tunneled staff for the latter proceeding. He regards it as an error to retain a catheter after either operation. We need not, by the way, touch on the controversial correspondence between our author and Professors Van Buren and Bumstead in regard to tunneled urethra instruments.

In discussing the subject of Retention of Urine, the author refers to the old practice of treating retention due to inflammatory swelling of the urethra in the following terms (page 217): "In former times, patients suffering in this way were treated with opiates, hot baths, and leeches to the perineum, all of which are very good, but are certainly insufficient to relieve the present distress. Prompt and efficient succour is now given in these cases by the immediate use of the catheter, without any damage being done to the urethra, and is accomplished by the cautious introduction of a well-oiled No. 6 or 7 gum catheter." Somewhat energetic attempts have been made of late to revive the practice here condemned, but the published results are not such as to recommend it.

Affections of the prostate are very briefly treated; and so also is the subject of stone in the bladder. The only novelty is the last chapter, which is devoted to a description, largely illustrated, of the method of "perineal lithotrity" recommended by M. Dolbeau, of Paris. Dr. Gouley seems to have a strong preference for this method, which, however, can be but rarely required. It consists in opening the membranous urethra from the perineum, and then dilating the prostate and neck of the bladder with a special instrument; after which a strong forceps or lithoclast is introduced into the bladder, and the calculus broken into fragments sufficiently small to be readily extracted. Professor Dolbean seems to have had thirty operations, with five deaths; and Dr. Gouley has had three successful cases. This practice would appear to be in direct opposition to the modern views respecting the non-dilatability of the neck of the bladder, and the necessity for free incisions through the prostate.

Practical Medicine, with a Sketch of Physiology and Therapeutics. By Alexander Silver, M.A., M.D. London: Henry Renshaw.

WHILE adopting the grouping of physiology, pathology, and therapeutics, Dr. Silver asks that his new work on the old basis of "Meade's Manual" may be judged as a book on Practice of Medicine.

As regards the part on Physiology, we need only point to the page (34) on Bodily Heat, to show what a narrow line divides condensation from confusion. The student who accepts the normal temperature as $100^{\circ}$, and believes that "by adding to the diet oily substances" and "by wearing warm clothing" he can in a healthy individual effect an increase of bodily heat, will find his pbysiological experiments somewhat at fault.

The part on Therapeutics, being but the epitome of an ordinary materia medica, will be an unwelcome substitute for the list of useful formulæ which are usually appended to handbooks on medicine. 